\title{
An Intelligent Methods for Pointer Instrument Reading Based on Machine Vision
}

\author{
Huanjun Liu \\ Faculty of Automation, Guangdong University of Technology, Guangzhou, Guangdong, China
}

\begin{abstract}
This paper puts forward to a novel intelligent methods for pointer instrument reading to replace human reading. The images of pointer instrument are captured. Firstly a segmentation algorithm based on unsupervised learning is presented to segment the image for locating the scales and pointer. This algorithm combines the fuzzy $\mathrm{C}$-means method with fuzzy support vector machines, and can subdivide the image into the objects and background efficiently. Secondly the according to the characteristic of pointer instrument, the central of instrument pointer is confirmed by Hough transform. And then the central angles of the scale regions and pointer region are calculated. At last the reading value of pointer instrument is determined. The Experiments prove the method presented by this paper is effective.
\end{abstract}

Keywords-pointer instrument reading; machine vision; unsupervised learning

\section{INTRODUCTION}

At present, the pointer instrument is widely used in the producing process. Generally, people read the pointer instrument by human eyes. But the human reading need a great deal of human resources, and is not stability. Some research has been done on the automatic reading the pointer instrument ${ }^{[1,2]}$. This paper presents a novel method based on machine vision to calculate the reading value of pointer instrument.

\section{Scales And Pointer REgions Segmentation}

The image of pointer instrument is shown in figure 1 . If the pointer instrument want to be read, the scales and the pointer must be located. So the regions of scales and pointer in the image need be segmented. But because the material of dial and pointer is different, and the glass covers the dial, the gray level of instrument image is not uniform.

To segment the image, a novel algorithm based on unsupervised learning is presented. Clustering can be used to divide a digital image into distinct regions for border detection or object recognition ${ }^{[3,4]}$, but the performance are not satisfying. This paper puts forward to a novel methods based on unsupervised pattern classification. This method firstly uses Fuzzy C-means methods to classify the image, and then chooses the samples which is high-confidence by fuzzy voting. These samples are used to train the fuzzy support vector machines. Finally the image is classified by trained fuzzy SVM.

The fuzzy c-means objective function $\left\{x_{K}\right\}_{k=1}^{N}$ for partitioning into clusters is given by:

$$
J=\sum_{i=1}^{c} \sum_{k=1}^{N} u_{i k}^{p}\left\|x_{k}-v_{i}\right\|
$$

where $\left\{v_{i}\right\}_{i=1}^{c}$ are the prototypes of the clusters and the array $\left[v_{i k}\right]=U$ represents a partition matrix, $U \in u$, namely

$$
u\left\{u_{i k} \in[0,1] \mid \sum_{i=1}^{c} u_{i k}=1 \forall k 0<\sum_{k=1}^{N} u_{i k}<N \forall i\right\}
$$

The parameter is a weighting exponent on each fuzzy membership and determines the amount of fuzziness of the resulting classification. The FCM objective function is minimized when high membership values are assigned to voxels whose intensities are close to the centroid of its particular class, and low membership values are assigned when the voxel data is far from the centroid.

Because the pixels in image are not isolated points, the features which can represent the characters of region are chose. The three features are used for FCM, which are as follow.

The feature $f s_{1}(x, y)$ of point $(\mathrm{x}, \mathrm{y})$ is :

$$
f s_{1}(x, y)=\operatorname{gray}(x, y)
$$

where gray $(x, y)$ is the gray level of point $(x, y)$.

The feature $f_{S_{2}}(x, y)$ is:

$$
f S_{2}(x, y)=\frac{\sum_{i=-1}^{1} \sum_{j=-1}^{1} \operatorname{gray}(x+i, y+j)}{9}
$$

where gray $(\mathrm{x}, \mathrm{y})$ is gray level of point $(\mathrm{x}, \mathrm{y}) . f_{s_{2}}(x, y)$ is average gray level of the 9 point in neighborhood of point (x,y).

The feature $f_{S_{3}}(x, y)$ is: 


$$
\begin{aligned}
f s_{3}(x, y) & =\operatorname{Var}(X) \\
& =\frac{\sum_{i=-1}^{1} \sum_{j=-1}^{1}(\operatorname{gray}(x+i, y+j)-\mu)^{2}}{9}
\end{aligned}
$$

where gray(x,y) is gray level of point $(\mathrm{x}, \mathrm{y})$,

$$
\mu=\frac{\sum_{i=-1}^{1} \sum_{j=-1}^{1} \operatorname{gray}(x+i, y+j)}{9} .
$$

After classifying the image by FCM according to the features, the high-confidence samples are chose by following condition. First the membership value of the sample is larger than a threshold:

$$
f m(x, y)>T_{m}
$$

where $f m(x, y)$ is a membership value of point $(\mathrm{x}, \mathrm{y})$ which is assigned by FCM, and the $T_{m}$ is a threshold which is 0.8 in this paper.

Second condition is that there are 3 points in neighborhood of the sample which also meets the first condition.

After choosing the samples, these samples are used to train the fuzzy support vectors. SVMs have been used as one of the highest performance classifying systems because of their ability to generalize well. In this paper, fuzzy support vector machines are presented. It combines fuzzy theory with SVMs.

FSVM consists of fuzzy layer and SVMs. Fuzzification is the function of the fuzzy layer. The construction of the fuzzy support vector machine is shown in figure 1.

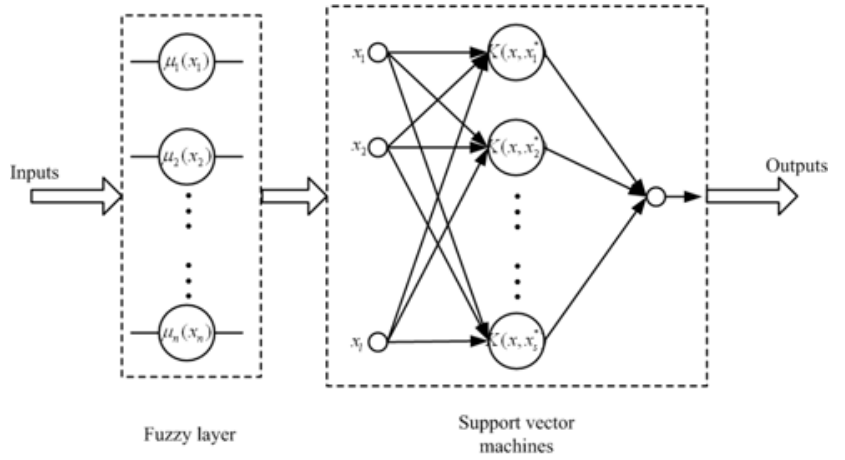

FIGURE I. THE CONSTRUCTION OF FUZZY SUPPORT VECTOR MACHINE

The features are inputted into the fuzzy layer, and translated into fuzzy outputs. This layer uses Gaussian function as the membership function. The function is as follows:

$$
\mu_{i}\left(x_{i}\right)=\mathrm{e}^{-\left(\frac{x_{i}-a_{i}}{b_{i}}\right)^{2}}
$$

Then, SVMs are used as the classifier for fuzzy outputs.

Research shows that the use of the hybrid kernel yields a better performance than those with a single common kernel ${ }^{[5]}$. Hence, the hybrid kernel is applied in this study. The kernel function adopted in this paper is as follows:

$$
K\left(x, x_{i}^{*}\right)=k_{1}\left(x \cdot x_{i}^{*}\right)^{d}+k_{2} \mathrm{e}^{-r\left|x-x_{i}^{*}\right|^{2}}
$$

Genetic algorithms (GAs) constitute the global optimization techniques known to be successful in many domains. Thus, a GA based selection of components for FSVM is proposed in this study. This method is employed to optimize FSVM. The accuracy of classification are used to evaluate the performance of classification.

The segment results are shown in figure 2.

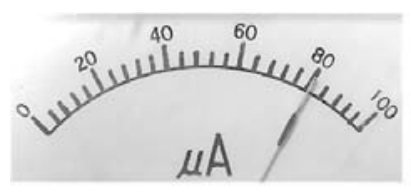

(1)

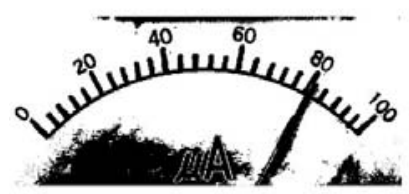

(3)
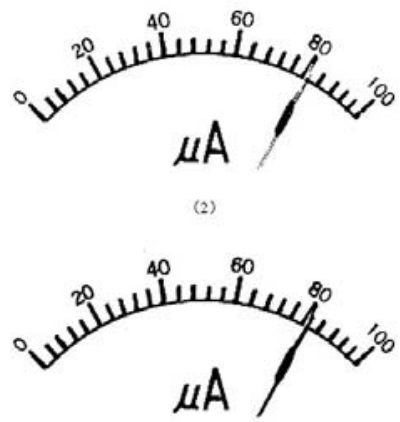

FIGURE II. THE SEGMENT RESULTS

The figure2(1) is the original image, the figure2(2) is segmented by Otsu method, the figure2(3) is the segmented by Tsallis entropy, and the figure2(4) is segmented by the methods presented in this paper.

\section{Reading Value Being Calculated}

After segmenting, the regions of the scales and painter are known. According to the characters of painter instrument, the scales and painter are ranged around a center. The Hough transform is used to identify this center. The edge below the scale region is a circle arc, whose center is also the center of all region. The center and radius of this circle arc can be get by Hough transform. The center and radius can be calculated by the three points on the edge. If the enough permutations of the three points are used to calculate the center and the radius, the real center and radius can be identified by maximum probability. After identifying the center and radius, because the connected region of scales is a part of circular ring, it can be removed by this method. If the point belongs to the region which meet the condition like equation (9), it can be removed. 


$$
\sqrt{\left(x-x_{C}\right)^{2}+\left(y-y_{C}\right)^{2}} \leq R_{C}+T_{C}
$$

which $(x, y)$ is the coordinate of the point, $\left(x_{C}, y_{C}\right)$ is the coordinate of the center, the $R_{C}$ is the radius, and the $T_{C}$ is a threshold.

Because the main part of the pointer is below the connected region, the pointer region can be identified by the similar method. If these point are removed, the remaining part is the pointer region.

$$
\sqrt{\left(x-x_{C}\right)^{2}+\left(y-y_{C}\right)^{2}} \geq R_{C}+T_{C}
$$

The instrument reading can be got by comparing the central angle of the painter with the central scale regions. So the central angle of every scale need be obtained. The axis of a scale region can be got. The lines between center of the all regions and the starting point, midpoint and ending point of axis are made, and the angle between each lines and the $\mathrm{X}$-axis is calculated. The angle of the scale is determined by the formula 11

$$
A S_{n}=\frac{\sum_{i=1}^{3} A L_{i}}{3}
$$

where $A L_{i}$ is the angle between the lines and the $\mathrm{X}$-axis, it is given by $A L_{i}=\arctan \left(\left(y_{i}-y_{C}\right) /\left(x_{i}-y_{C}\right)\right)$.

The central angle of the painter region, $A P$, is got by similar method.

The some part of the painter is above the connected region, so this region may be mistakenly taken as the scale region. For removing this region, the method is adopt. If the region meet the two conditions, the scale region $\mathrm{n}$ is taken for the part of pointer. The condition 1 is:

$$
\left|A S_{n}-A P\right| \leq \varepsilon
$$

where AP is the central angle of the painter, $A S_{n}$ is the central angle of the scale region n, $\varepsilon$ is a small threshold.

The condition 2 is:

$$
\begin{gathered}
A S_{n}-A S_{n-1}<A S_{n-1}-A S_{n-2} \\
\text { and } A S_{n+1}-A S_{n}<A S_{n+2}-A S_{n+1} \\
\text { Or } A S_{n}-A S_{n-1}<\left(A S_{n-1}-A S_{n-2}\right) / 2 \text {; } \\
\text { Or } A S_{n+1}-A S_{n}<\left(A S_{n+2}-A S_{n+1}\right) / 2 .
\end{gathered}
$$

where $A S_{n}$ is the central angle of the scale region $\mathrm{n}$.

3.

The scale regions and the pointer region are shown in figure

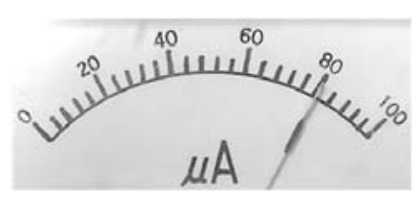

(1)

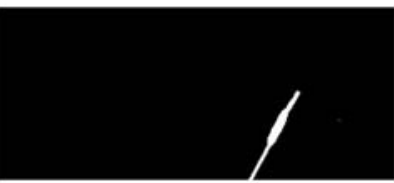

(3)

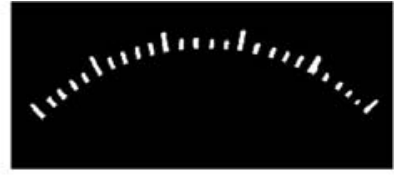

$(2)$
FIGURE III. THE SCALE AND POINTER REGIONS

The figure 3(1) is the original image, the figure 3(2) is the scale regions, and the figure $3(3)$ is the pointer region.

If the painter is between scale $K$ and scale $K+1$, the instrument reading is calculated by formula 13 .

$$
S R=\frac{S V_{K+1}-S V_{k}}{A S_{K+1}-A S_{k}}\left(A P-A S_{k}\right)
$$

where $S V_{K}$ is the value of the scale $\mathrm{K}$, the $A S_{K}$ is the central angle of the scale $\mathrm{K}$, and the AP is the central angle of the painter.

\section{EXPERIMENT}

The 100 images of painter instrument are captured. The reading value of each image is first given by human. Then the reading values of these instrument are calculated by the methods presented in this papers. The comparison shows that calculated reading values are in agreement with the readings given by human within a margin of error.

\section{CONCLUSION}

This paper presents a novel intelligent method based on machine vision to calculate the reading value of pointer instrument. After capturing image, the regions of scales and pointer in the image need be segmented. But the gray level of instrument image is not uniform, so a novel segment algorithm based on unsupervised learning is presented. This algorithm combines the fuzzy C-means method with fuzzy support vector machines, and can subdivide the image into the objects and background efficiently. Because the scales and painter are ranged around a center of dial, the Hough transform is used to identify this center. And then the scale and pointer regions are extracted separately. The central angles of the scale regions and pointer region are calculated. At last the reading value of pointer instrument is determined by compare the central angle of pointer and the scales. The Experiments shows the calculated reading values are in agreement with the readings given by human within a margin of error. 


\section{REFERENCES}

[1]. GUO Yong-cai, LIU Li-jun, GAO Chao. Automatic reading system of meter based on image sensor. Transducer and Microsystem Technologies, Vol.32 pp.101-105, 2012

[2]. XU Yang, LIAO Qinyu, LI Rui, CHENG Anyu .Research and Design of Vision Inspection System of Automobile Pointer Instrument. Video Engineering, Vol. 36 pp 83-88, 2012

[3]. Zhang Y, Fu-Lai Chung, Wang S T. Robust fuzzy clustering based image segmentation. Applied Soft Computing, Vol. 9, pp.80-84, 2009.

[4]. YangM-S, TsaiH-S. A Ganssian kernel-based fuzzy c-means algorithm with a spatial bias correction. Pattern Recognition Letters, Vol.29, pp.1713-1725, 2008.

[5]. Tan Y, Wang J. A support vector machine with a hybrid kernel and minimal Vapnik-Chervonenkis dimension. IEEE Transactions on Knowledge and Data Engineering, Vol.4, pp. 385-295, 2004. 